\title{
Gastric Neuroendocrine Tumor Mimicking Gastrointestinal Stromal Tumor: A Case Report
}

\author{
Jin Lee ${ }^{a}$ Sung Jin Oh ${ }^{b}$ \\ aDepartment of Internal Medicine, Inje University Haeundae Paik Hospital, Inje University \\ College of Medicine, Busan, Republic of Korea; ${ }^{b}$ Department of Surgery, Inje University \\ Haeundae Paik Hospital, Inje University College of Medicine, Busan, Republic of Korea
}

\section{Keywords}

Subepithelial tumor · Neuroendocrine tumor · Gastrointestinal stromal tumor

\begin{abstract}
Gastric neuroendocrine tumors (NETs) are rare lesions that arise from enterochromaffin-like cells of the gastric mucosa. Gastric NETs are classified into 3 types of NETs and poorly differentiated neuroendocrine cancers. Most gastric NETs usually present as hemispherical, yellowish, polypoid lesions with a central depression and often as subepithelial tumors (SETs) because they are confined within the submucosal layer. Here, we report a case of gastric NET presenting as SET mimicking a gastrointestinal stromal tumor (GIST). Endoscopy revealed a 2.3-cm-sized SET with intact surface mucosa, and endoscopic ultrasonography showed a homogeneous hypoechoic lesion with a well-circumscribed margin. Typical features of gastric NET, such as yellowish mucosal changes or central ulceration, were not observed. GIST was suspected, and a laparoscopic wedge resection was performed. The final diagnosis was gastric NET with grade 2 differentiation.
\end{abstract}

\section{Introduction}

Gastric neuroendocrine tumors (NETs) are characterized by enterochromaffin-like cells of the stomach and are relatively rare tumors, accounting for 7\%-9\% of all gastrointestinal NETs [1, 2]. Gastric NETs are divided into 3 types of NETs and poorly differentiated neuroendocrine cancers based on the clinicopathological features, and identification of the type is important for the diagnosis, treatment, and prediction of the disease prognosis [3]. Histologic diagnosis of gastric NET is important because the treatment strategy is determined based on 

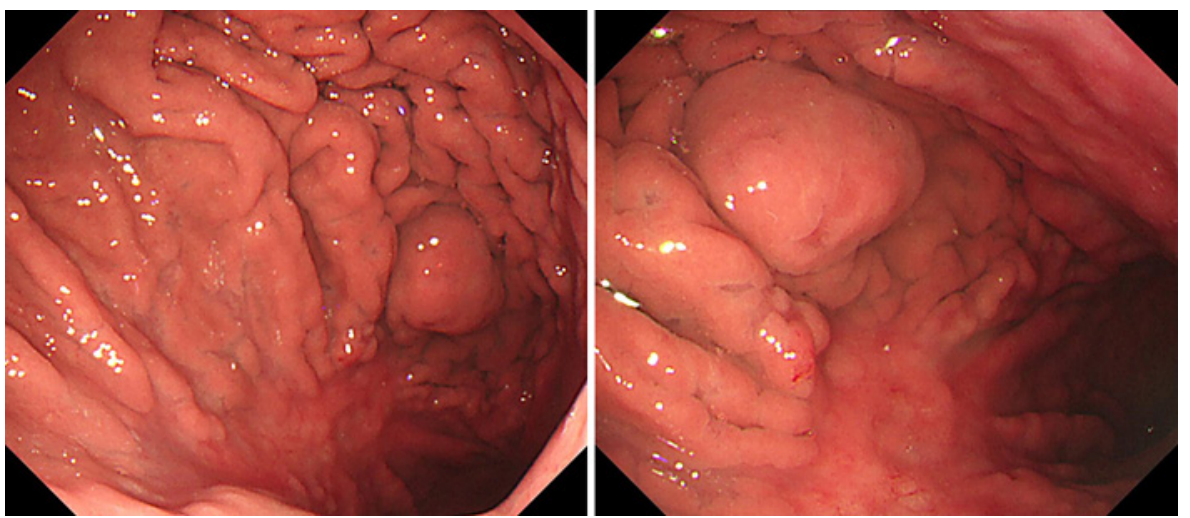

Fig. 1. Endoscopy showing a 2-cm lobulated lesion with normal mucosa at the greater curvature of the midbody of the stomach.

the tumor type, tumor size, tumor grade, and the presence of metastasis [4]. Most gastric NETs usually present as hemispherical, yellowish, polypoid lesions with a central depression and often as subepithelial tumors (SETs). Gastric NETs with a submucosal form are difficult to confirm histologically and to distinguish from gastrointestinal stromal tumors (GISTs) or other diseases. Herein, we report a rare case of gastric NET that mimicked GIST, which was diagnosed after laparoscopic wedge resection.

\section{Case Presentation}

A 66-year-old man visited our hospital for a screening endoscopy. His vital signs were stable, and physical examination was unremarkable on initial outpatient examination. Laboratory test results were unremarkable, with a normal range of serum carcinoembryonic antigen and carbohydrate antigen 19-9 levels. Endoscopy revealed an approximately 2.3-cm-sized gastric SET with normal overlying mucosa at the greater curvature of the mid-body of the stomach (Fig. 1). Application of pressure with forceps showed a positive rolling sign and a negative cushion sign. Biopsy was performed from a sample from the mucosal surface of the SET, and the report showed gastritis with no submucosal component. Endoscopic ultrasonography (EUS) with a linear ear endoscope was performed to evaluate the characteristics of the tumor. EUS revealed an approximately $23 \times 16 \mathrm{~mm}$ homogeneous hypoechoic lesion with a well-defined margin that was thought to arise from the fourth layer (muscularis layer) (Fig. 2). 3-Dimensional computed tomography (CT) of the stomach showed a 2.3-cm-sized homogeneous enhancing lesion on the greater curvature of the gastric mid-body (Fig. 3). There was no evidence of lymph node enlargement or distant metastasis. GIST was clinically suspected based on the findings of preoperative imaging tests, including endoscopy, EUS, and CT.

Histological confirmation or resection was recommended because the tumor was more than $2 \mathrm{~cm}$ in size, which is considered a high-risk feature. EUS-guided needle biopsy (FNB) is recommended for an accurate diagnosis; however, the patient preferred a laparoscopic wedge resection for simultaneous histological confirmation and treatment. Laparoscopic wedge resection was performed after consultation with the patient. After wedge resection, gross examination showed a protruding mass measuring $2.3 \times 2.0 \mathrm{~cm}$, and the cut sections revealed a well-defined and yellowish mass infiltrating the submucosal layer. The histopathological results were as follows (Fig. 4): the arrangement of the tumor cells formed solid nests 


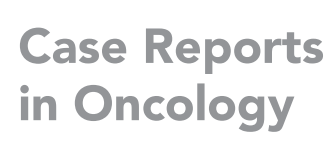

\begin{tabular}{l|l}
\hline Case Rep Oncol 2021;14:1271-1276 \\
\hline DOI: 10.1159/000518078 & $\begin{array}{l}\text { ○ 2021 The Author(s). Published by S. Karger AG, Basel } \\
\text { www.karger.com/cro }\end{array}$ \\
\hline
\end{tabular}

Lee and Oh: Gastric NET Mimicking GIST


Fig. 2. EUS shows an approximately $23 \times 20 \mathrm{~mm}$ homogeneous hypoechoic lesion with a well-circumscribed margin. EUS, endoscopic ultrasound.
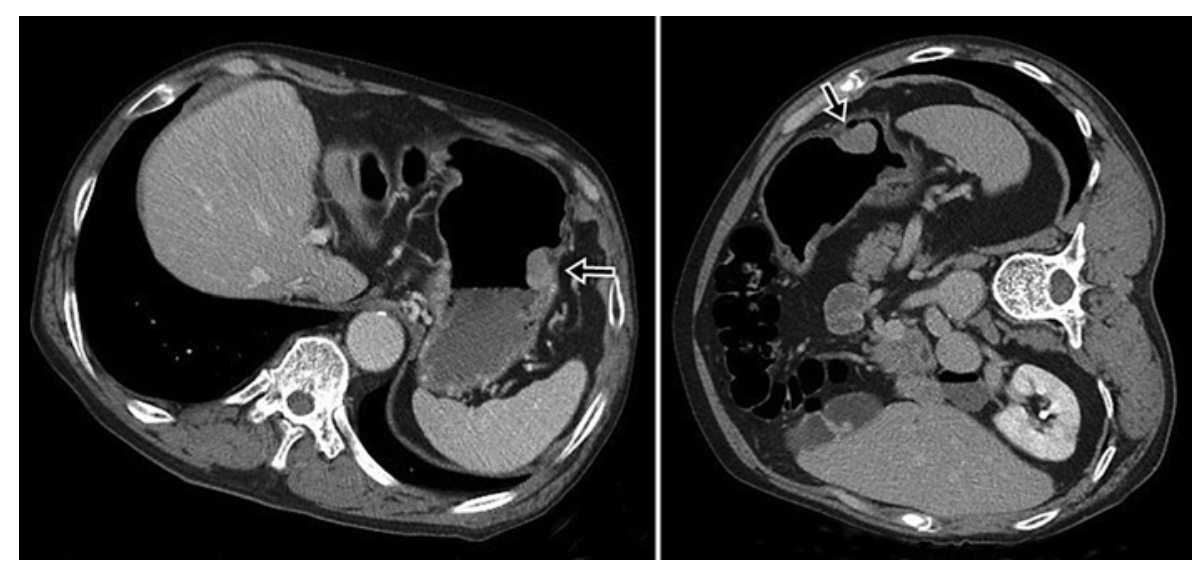

Fig. 3. 3D CT of the stomach shows a $2.3-\mathrm{cm}$-sized homogeneous enhancing lesion (arrow) on the greater curvature of the gastric mid-body. CT, computed tomography.

or sheet-like structures in the submucosal layer with intact mucosa and the muscularis layer. Immunohistochemical evaluation revealed immunopositivity for chromogranin A and synaptophysin, and immunonegativity for CD56. The Ki-67 labeling index was determined to be $15 \%$. The serum gastrin level was increased to $419.0 \mathrm{pg} / \mathrm{mL}$ (normal range: $13-115 \mathrm{pg} / \mathrm{mL}$ ), and atrophic change was detected on endoscopy. Thus, the final diagnosis was a type 1 NET grade 2 based on the clinicopathological features. Follow-up endoscopy and CT were scheduled after 6 months.

\section{Discussion and Conclusion}

Gastric NETs, previously known as carcinoid tumors, are characterized by enterochromaffin-like cells of the stomach. In recent times, the incidence of gastric NETs has increased, which might be due to better clinical awareness and an increase in screening endoscopy. Gastric NETs are classified into 3 types of NETs and poorly differentiated neuroendocrine cancers based on their clinicopathological features [5]. Type 1 is associated with chronic atrophic gastritis. Type 2 is observed in patients with type 1 multiple endocrine neoplasia. Type 3 is a sporadic tumor with no specific underlying diseases. A poorly differentiated 


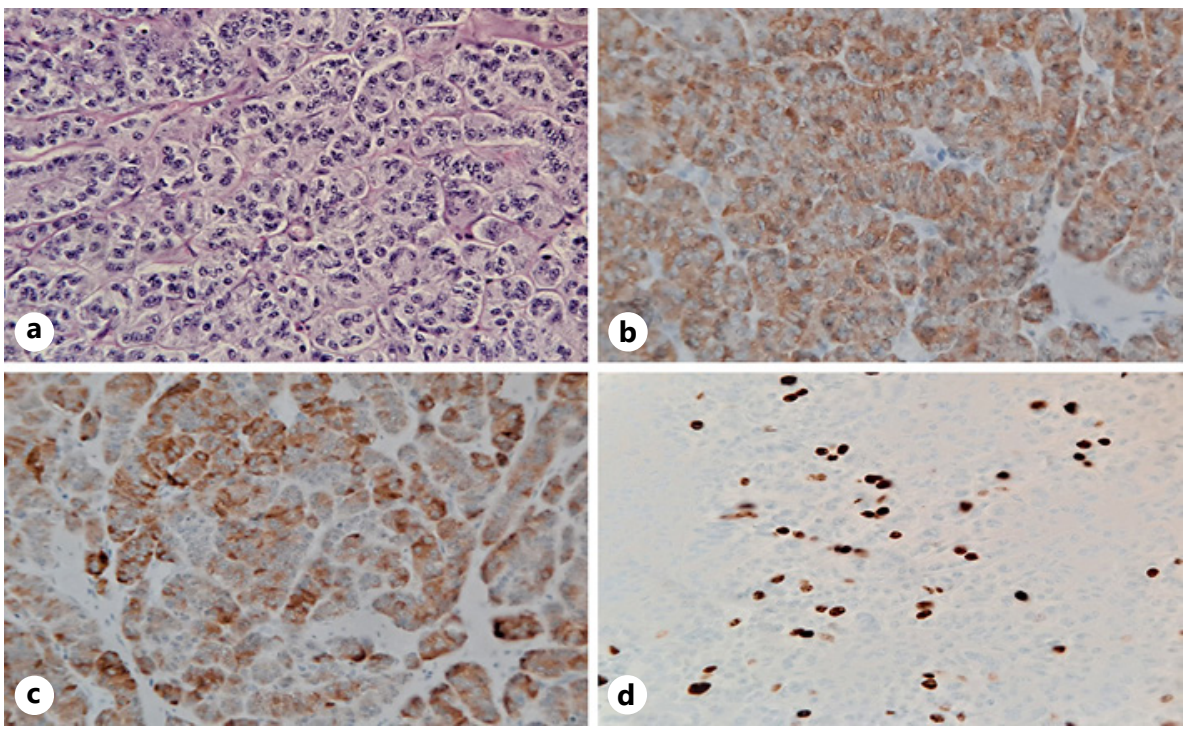

Fig. 4. Histopathology findings. Hematoxylin and eosin staining of the lesion (a); Immunohistochemistry revealed positivity for chromogranin A (b); Immunohistochemistry revealed positivity for synaptophysin (c); Positively stained cells were found for Ki-67 and a proliferation index of 15\% was evident on immunohistochemistry (d).

neuroendocrine cancer is referred to as type 4 NET. Type 1 and 2 NETs usually manifest as multiple lesions $\leq 1 \mathrm{~cm}$ in size, and type 3 NETs usually appear as a single lesion $\geq 2 \mathrm{~cm}$ in size.

Most gastric NETs usually present as yellowish sessile or polypoid lesions with an occasional central depression or ulceration, and sometimes as a SET because they are confined within the submucosal layer. Type 1 gastric NETs usually appear as multiple polyps in the fundus of the stomach. Typical EUS findings show hypoechoic or isoechoic homogeneous lesions with distinct margins in the second or third layer of the stomach. Compared with other SETs, the yield of routine endoscopic biopsy of gastric SET is relatively high, although the mass is covered with normal mucosa. In this case, there were no typical endoscopic findings of NET, such as a sessile or polypoid yellowish lesion with a central depression. Moreover, it was difficult to confirm the histology using routine endoscopic biopsy. Since the SET was more than $2 \mathrm{~cm}$ in size, which is a high-risk feature, histologic confirmation or resection was necessary. Various methods, such as EUS-guided fine needle aspiration, EUS-FNB, endoscopic resection, and surgical approach, are used for histologic confirmation [6]. In many cases, a surgical approach, such as laparoscopic wedge resection, is considered useful for the treatment of gastric SETs because simultaneous diagnosis and treatment are possible.

In this case, EUS-FNB was recommended for an accurate diagnosis; however, the patient preferred laparoscopic wedge resection for simultaneous histological confirmation and treatment. Histopathological analysis of the resected specimen showed NET grade 2. The diagnosis was type 1 NET because a definite atrophic change was detected on endoscopy, and serum gastrin level was increased. Histopathological evaluation of the resected specimen showed that the tumor had invaded the submucosa, while the mucosa and muscularis were intact. It might be misdiagnosed on preoperative EUS as SET arising from the fourth layer. Although EUS is considered a useful diagnostic method for gastric SETs, EUS has a low accuracy of $45 \%-65 \%[7,8]$ and sometimes, the layer of the origin or internal echogenicity of the SET might not be clear and distinct on EUS. In the case of gastric SET with indistinct characteristics on EUS, additional treatment might be necessary after confirmation of the histologic diagnosis based on the resected specimen.

\section{Karger'}


In patients with gastric NETs, the therapeutic approach depends on the tumor subtype, grade of differentiation, and extent of invasion. Poor prognostic factors include size of $\geq 2 \mathrm{~cm}$, submucosal or muscularis invasion, a Ki-67 index of $\geq 3 \%$, the presence of dysplasia or necrosis, and a low degree of differentiation [9]. According to the National Comprehensive Cancer Network guidelines [10], endoscopic resection or surgery is recommended for type 1 and 2 gastric NETs $\geq 2 \mathrm{~cm}$ in size. Surgical treatment should be considered if there is a positive margin after initial resection or if there are poor prognostic factors. Although antrectomy has been used as a surgical treatment method in the past, laparoscopic wedge resection is currently performed for single lesions, and more extensive surgery can be considered in cases of $>6$ lesions or recurrence [11]. However, there is still controversy about whether surgery is the best treatment option for NETs with poor prognostic features. A recent meta-analysis showed that local recurrence occurred in $22 \%$ of the patients after endoscopic resection; however, there was no statistical difference compared with surgical treatment, and the 5 -year disease-specific survival rate was reported to be $100 \%$ regardless of lymph node metastasis [12]. In the present case, since a GIST was clinically suspected based on the preoperative imaging test results and there were no typical findings of NET on endoscopy, laparoscopic wedge resection was performed, and the patient was followed up after completion of treatment. Close observation is necessary through regular follow-up endoscopy and CT because of the presence of features associated with poor prognosis: a size of $\geq 2 \mathrm{~cm}$ and a Ki-67 index of $\geq 3 \%$.

Overall, this case suggests that gastric NET can present as a round SET with intact mucosa, mimicking GIST. In recent times, although preoperative histologic diagnosis using EUS-FNB and endoscopic resection has been reported to be useful in the management of gastric SET, laparoscopic wedge resection is desirable for simultaneous diagnosis, treatment, and preservation of the stomach. In the case of gastric SETs with high-risk features, especially those that are $\geq 2 \mathrm{~cm}$ in size, it is necessary to determine the appropriate treatment strategy.

\section{Acknowledgment}

We appreciate the patient for allowing the presentation of this case.

\section{Statement of Ethics}

Written informed consent for the publication of the patient's clinical details and images was obtained from the patient. No institutional review board or other approvals were required since it is de-identified.

\section{Conflict of Interest Statement}

The authors have no conflicts of interest to declare.

\section{Funding Sources}

The authors declared that this study has received no financial support.

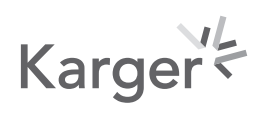




\section{Author Contributions}

Jin Lee drafted the manuscript; Sung Jin Oh revised the manuscript; Jin Lee and Sung Jin Oh were involved in the conception and design of the case report.

\section{Data Availability Statement}

All data including patient's clinical details and images are included in this article.

\section{References}

1 Modlin IM, Lye KD, Kidd M. A 5-decade analysis of 13,715 carcinoid tumors. Cancer. 2003;97(4):934-59.

2 Modlin IM, Lye KD, Kidd M. A 50-year analysis of 562 gastric carcinoids: small tumor or larger problem? Am J Gastroenterol. 2004;99(1):23-32.

3 Li TT, Qiu F, Qian ZR, Wan J, Qi XK, Wu BY. Classification, clinicopathologic features and treatment of gastric neuroendocrine tumors. World J Gastroenterol. 2014;20(1):118-25.

4 Park MI. Endoscopic treatment for early foregut neuroendocrine tumors. Clin Endosc. 2013;46(5):450-5.

5 Rindi G, Luinetti O, Cornaggia M, Capella C, Solcia E. Three subtypes of gastric argyrophil carcinoid and the gastric neuroendocrine carcinoma: a clinicopathologic study. Gastroenterology. 1993;104(4):994-1006.

6 Cho JW, Korean ESD Study Group. Current guidelines in the management of upper gastrointestinal subepithelial tumors. Clin Endosc. 2016;49(3):235-40.

7 Karaca C, Turner BG, Cizginer S, Forcione D, Brugge W. Accuracy of EUS in the evaluation of small gastric subepithelial lesions. Gastrointest Endosc. 2010;71(4):722-7.

8 Lim TW, Choi CW, Kang DH, Kim HW, Park SB, Kim SJ. Endoscopic ultrasound without tissue acquisition has poor accuracy for diagnosing gastric subepithelial tumors. Medicine. 2016;95(44):e5246.

9 Dias AR, Azevedo BC, Alban LBV, Yagi OK, Ramos MFKP, Jacob CE, et al. Gastric neuroendocrine tumor: review and update. Arq Bras Cir Dig. 2017;30(2):150-4.

10 Kulke MH, Shah MH, Benson AB 3rd, Bergsland E, Berlin JD, Blaszkowsky LS, et al. Neuroendocrine tumors, version 1.2015. J Natl Compr Canc Netw. 2015;13(1):78-108.

11 Kaltsas G, Grozinsky-Glasberg S, Alexandraki KI, Thomas D, Tsolakis AV, Gross D, et al. Current concepts in the diagnosis and management of type 1 gastric neuroendocrine neoplasms. Clin Endocrinol. 2014;81(2):157-68.

12 Tsolakis AV, Ragkousi A, Vujasinovic M, Kaltsas G, Daskalakis K. Gastric neuroendocrine neoplasms type 1: a systematic review and meta-analysis. World J Gastroenterol. 2019;25(35):5376-87. 